\title{
Hyperthermic Intraperitoneal Chemotherapy
}

National Cancer Institute

\section{Source}

National Cancer Institute. Hyperthermic Intraperitoneal Chemotherapy. NCI Thesaurus. Code C91835.

A procedure performed in combination with abdominal surgery for cancer that has spread to the abdomen. It involves the infusion of a heated chemotherapy solution that circulates into the abdominal cavity. 